\title{
Heart rate variability predicts cell death and inflammatory responses to global cerebral ischemia
}

\author{
Greg J. Norman ${ }^{1}$, Kate Karelina ${ }^{2}$, Gary G. Berntson ${ }^{2}$, John S. Morris ${ }^{2}$, Ning Zhang ${ }^{3}$ and \\ A. Courtney DeVries ${ }^{3 *}$
}

${ }^{1}$ Center for Cognitive and Social Neuroscience, The University of Chicago, Chicago, IL, USA

${ }^{2}$ Department of Psychology, The Ohio State University, Columbus, OH, USA

${ }^{3}$ Department of Neuroscience, The Ohio State University, Columbus, OH, USA

\section{Edited by:}

Jacqueline Kathleen Phillips,

Macquarie University, Australia

Reviewed by:

Mathias Baumert, University of

Adelaide, Australia

Cara Hildreth, Macquarie University,

Australia

\section{*Correspondence:}

A. Courtney DeVries, Department of

Neuroscience, The Ohio State

University, 706 BRT, 460 12th Avenue

Columbus, OH 43210, USA.

e-mail: devries.14@osu.edu
This study examines the relationship between autonomic functioning and neuropathology following cardiac arrest (CA) in mice. Within $24 \mathrm{~h}$ of CA, parasympathetic cardiac control, as indexed by high frequency (HF) heart rate variability, rapidly decreases. By day 7 after CA, $\mathrm{HF}$ heart rate variability was inversely correlated with neuronal damage and microglial activation in the hippocampus. Thus, by virtue of its sensitivity to central insult, HF heart rate variability may offer an inexpensive, non-invasive method of monitoring neuropathological processes following $\mathrm{CA}$. The inverse linear relationships between heart rate variability and brain damage after CA also may partially explain why low heart rate variability is associated with increased morbidity and mortality in myocardial infarction patients.

Keywords: cardiac, autonomic, cardiac arrest, ischemia, parasympathetic

\section{INTRODUCTION}

Sudden cardiac arrest (CA) is a leading cause of mortality worldwide, affecting more than 800 individuals in The United States and 2,000 individuals in Europe every day (Sans et al., 1997; American Heart Association, 2002). While survival rates following CA remain staggeringly low, the individuals who do survive suffer from a myriad of pathological conditions resulting from neuronal damage associated with cessation of blood flow to the brain, otherwise known as global cerebral ischemia (Cronberg et al., 2009).

Ischemic brain injury resulting from CA is thought to exacerbate negative health status through a broad spectrum of mechanisms that include a diminished capacity to coordinate hormonal and autonomic processes important for metabolic, immune, and cardiovascular regulation (Micieli and Cavallini, 2008; Neigh et al., 2009; Offner et al., 2009; Tracey, 2009). Indeed, evaluation of neurocardiac functioning is used as a risk stratifier in patients with various cerebrovascular diseases (Thayer and Lane, 2007). A reduction in heart rate variability, a measure of parasympathetic cardiac control (Berntson et al., 1997), after myocardial infarction is associated with increased morbidity and mortality (Bigger et al., 1988; Huikuri et al., 2009; Karp et al., 2009). Despite the strong predictive value of heart rate variability in cardiovascular patients, the nature of the relationship between low heart rate variability and mortality is not well understood. Deficits in central regulation of heart rate variability represent one of the more likely explanations for the aforementioned relationship (Thayer and Lane, 2007). For example, lesions or pharmacological inhibition of higher level cortical structures are known to diminish parasympathetic cardiac regulation (Oppenheimer et al., 1996; Thayer et al., 2009). Therefore, measurement of neurocardiac functioning may provide a noninvasive proxy for alterations in the activity of higher level brain structures associated with ischemic damage.
The objective of the present study was to determine the effect of global cerebral ischemia on autonomic functioning in a mouse model. Furthermore, we tested the hypothesis that alterations in neurocardiac function covary with neuronal damage and inflammatory processes after CA.

\section{MATERIALS AND METHODS}

Adult male C57/BL6 mice (23-30 g; Charles River, Wilmington, MA, USA) were maintained on a 14:10 light/dark cycle and individually housed within a temperature and humidity-controlled vivarium. The study was conducted in accordance with NIH guidelines and approved by the OSU Institutional Animal Care and Use Committee.

\section{EXPERIMENTAL PROTOCOLS}

Animals were implanted with telemetric recording devices (ETAF20; DSI, St. Paul, MN, USA) for the determination of autonomic cardiac control, locomotor activity, and temperature. Animals were randomly assigned to two experimental groups: normothermic CA (CA/CPR; $n=12$ ) or hypothermic CA (control; $n=10)$.

\section{Cardiac arrest/CPR procedure}

As previously described (Neigh et al., 2009), mice were anesthetized with isoflurane, and then $8 \mathrm{~min}$ of CA was induced through injection of potassium chloride (KCL; $50 \mu \mathrm{l}, 0.5 \mathrm{M}, 4^{\circ} \mathrm{C}$ ) via a jugular catheter. $\mathrm{CPR}$ was initiated via injection of epinephrine (EPI; $16 \mu \mathrm{g}$ in $0.6 \mathrm{cc}$ saline, $37^{\circ} \mathrm{C}$ ) and chest compressions $(300 / \mathrm{min})$. A double lumen coil system was used to manipulate head temperature independently of body temperature. This was accomplished by having two independent coils (one for body and one for head) that were pumped with ice water to produce hypothermia in the area of interest. Temperature was continuously 
measured with thermometers located in the periphery (rectum) and the head temporalis muscle (which is highly correlated with brain temperature, Neigh et al., 2009). The periphery was maintained at $27^{\circ} \mathrm{C}$ during $\mathrm{CA} / \mathrm{CPR}$ to protect against peripheral organ damage. The heads of the CA/CPR mice were maintained at $37^{\circ} \mathrm{C}$ to allow the development of CA/CPR-induced neuroinflammation and neuronal damage, while the heads of the ischemic controls were maintained at $27^{\circ} \mathrm{C}$ to prevent the evolution of neurological damage (Neigh et al., 2009). Thus, the CA and ischemic control groups experience the same surgical preparations, duration of cerebral ischemia and exposure to KCL, EPI, and chest compressions, but only the CA/CPR group develops neuroinflammation and neuronal damage.

\section{Telemetry implant and heart rate variability analysis}

The mice were implanted with telemetric recording devices (DSI, St. Paul, MN, USA) at least 10 days prior to CA/CPR in accordance with previous studies (Thireau et al., 2008). The telemetric implants record temperature, activity, and electrocardiographic signals in the animal's home cage. The electrocardiographic signals are then subsequently used to derive $\mathrm{R}-\mathrm{R}$ intervals and heart rate variability. Mice were anesthetized with isoflurane and an ETA-F20 (Data Sciences International, St. Paul, MN, USA) telemetric probe was implanted into the abdominal cavity of the mouse and leads were placed onto opposite sides of the xyphoid process. Immediately following telemetric implants animals were treated with a local anesthetic to reduce pain (Bupivacaine). Commercial software (Mindware, Gahanna, OH, USA) was used to derive high frequency (HF) and low frequency (LF) heart rate variability, well validated measures of parasympathetic tone, in mice through spectral analysis of the interbeat interval series obtained from the ECG as measured by the telemetric implants (sampled at $1,000 \mathrm{~Hz}$ ). Analysis followed procedures recommended by the Society for Psychophysiological Research SPR committee on heart rate variability and was adapted for use in rodents (Berntson et al., 1997). Heart rate (R-R), temperature, and activity were sampled for $3 \mathrm{~min}$ at the beginning of every hour and analyzed in segments of $60 \mathrm{~s}$ and then averaged across the 3-min period for each hour. LF spectral power was integrated over $0.15-1.5 \mathrm{~Hz}$ and $\mathrm{HF}$ spectral power was integrated over the respiratory frequency band $(1.5-5 \mathrm{~Hz})$. The parameters used in the analysis of HF and LF heart rate variability were chosen based upon previous work in mice (Gehrmann et al., 2000; Thireau et al., 2008; Norman et al., 2010a).

\section{HISTOLOGY}

\section{Tissue collection}

At 7 days post-resuscitation, mice were deeply anesthetized and perfused transcardially with ice-cold $0.1 \mathrm{M}$ PBS and $4 \%$ paraformaldehyde. Brains were removed, post-fixed overnight in $4 \%$ paraformaldehyde, cryoprotected with $30 \%$ sucrose, and then frozen on dry ice. Brains were sectioned at $14 \mu \mathrm{m}$ on a cryostat and thaw-mounted onto Super Frost Plus slides (Fisher, Hampton, NH, USA). Histological images were captured with a Nikon E800 microscope (Nikon Instruments, Melville, NY, USA) and stored on a computer. Data collection was performed by an individual who was not informed of individual group assignments.

\section{Fluoro-Jade C histochemistry}

Tissue sections were stained according to established protocols (Schmued and Hopkins, 2000). The slides were dried at room temperature, immersed in a basic ethanol solution, and then rinsed in $70 \%$ ethanol and distilled water. Then, the slides were treated with potassium permanganate $(0.06 \%)$ for $10 \mathrm{~min}$, rinsed with water, incubated in Fluoro-Jade C $(0.0001 \%$ in a $1 \%$ acetic acid solution) then rinsed in $\mathrm{dH} 20$, dried on a slide-warmer, and cover-slipped with DPX (Sigma, St. Louis, MO, USA). FluoroJade positive cells were counted within the entire hippocampus and summed to form an aggregate measure of hippocampal neuronal damage; a structure known to be highly susceptible to global cerebral ischemic damage.

\section{MAC-/CD11b histochemistry}

Microglia were visualized using an antibody directed against MAC-1/CD11b as previously described (Popovich et al., 1997). Slides were air dried, rinsed in distilled water, and then blocked with rabbit serum and bovine serum albumin. Slides were incubated for $24 \mathrm{~h}$ at room temperature with rat anti-CD11b antibody (Serotec, Raleigh, NC, USA) diluted 1:100 in phosphate buffer. Slides were then rinsed and incubated with rabbit anti-rat secondary antibody (1:500; Vector Labs, Burlingame, CA, USA) for $2 \mathrm{~h}$. Sections were quenched in $\mathrm{H} 202$ in methanol and then rinsed and treated with Elite $\mathrm{ABC}$ reagent for $60 \mathrm{~min}$. Sections were visualized with 3,3'-diaminobenzidine containing nickel. After visualization, the slides were rinsed in distilled water, then dehydrated, cleared, and cover-slipped. Finally, proportional area of stained vs. non-stained areas were quantified within the entire hippocampus using image J software (NIH, Bethesda, MD, USA).

\section{Statistical analysis}

The data are expressed as means \pm SE of the mean. Testing of statistical significance was performed using ANOVA. Group differences were considered statistically significant at $p<0.05$. Autonomic, locomotor, and temperature responses were analyzed using twoway repeated measures. Spectral analysis was used to determine diurnal rhythms in heart rate variability, temperature, and locomotor activity. Pearson correlation coefficients were carried out between measures autonomic control and levels of Mac-1 and Fluoro-Jade C expression.

\section{RESULTS}

As expected, global cerebral ischemia resulted in significant increases in cell death $\left(F_{1,20}=17.73, p<0.01\right.$; Figure 1A $)$ and microglia responses $\left(F_{1,20}=10.47, p<0.01\right.$; Figure 1B $)$ within the hippocampus as compared to the ischemic control. Furthermore, the $\mathrm{CA} / \mathrm{CPR}$ procedure engendered significant decreases in $\mathrm{HF}$ heart rate variability $\left(F_{7,140}=16.31, p<0.05\right.$; Figure $\left.2 \mathrm{~A}\right)$, a well validated measure of parasympathetic cardiac control (Berntson et al., 1997). The CA/CPR procedure also resulted in a significant decline in LF heart rate variability $\left(F_{7,140}=12.08, p<0.05\right)$. Similarly, cerebral ischemia resulted in a significant increase in heart rate $\left(F_{7,140}=9.19, p<0.05\right.$; Figure $\left.2 \mathbf{B}\right)$. However, while the overall values of $\mathrm{HF}$ heart rate variability, LF heart rate variability, and heart rate were lower in CA/CPR animals than ischemic controls, a 2 (light vs. dark) $\times 2(\mathrm{CA} / \mathrm{CPR}$ vs. Control) ANOVA revealed that 

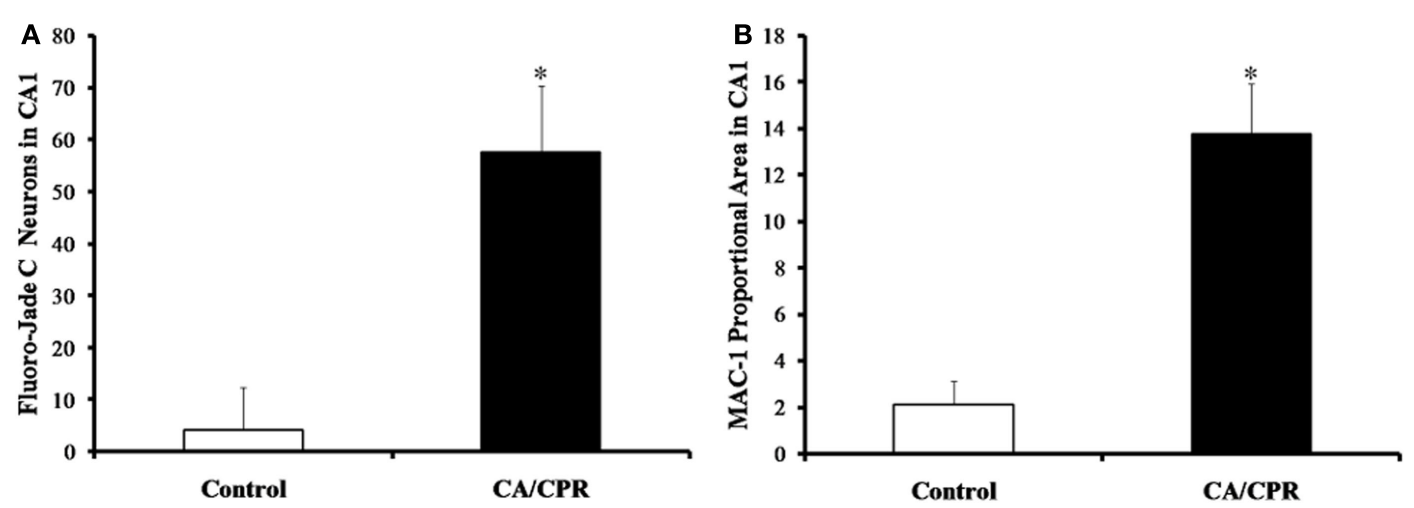

FIGURE 1 |The CA/CPR procedure increased levels of hippocampal cell death (A) and microglial expression (B) at 7 days following CA, as measured by Fluoro-Jade $\mathbf{C}$ and MAC-1 staining, respectively. The data are presented as mean $\pm \mathrm{SD}$ and an asterisk indicates a significant difference vs. control at $p<0.05$.
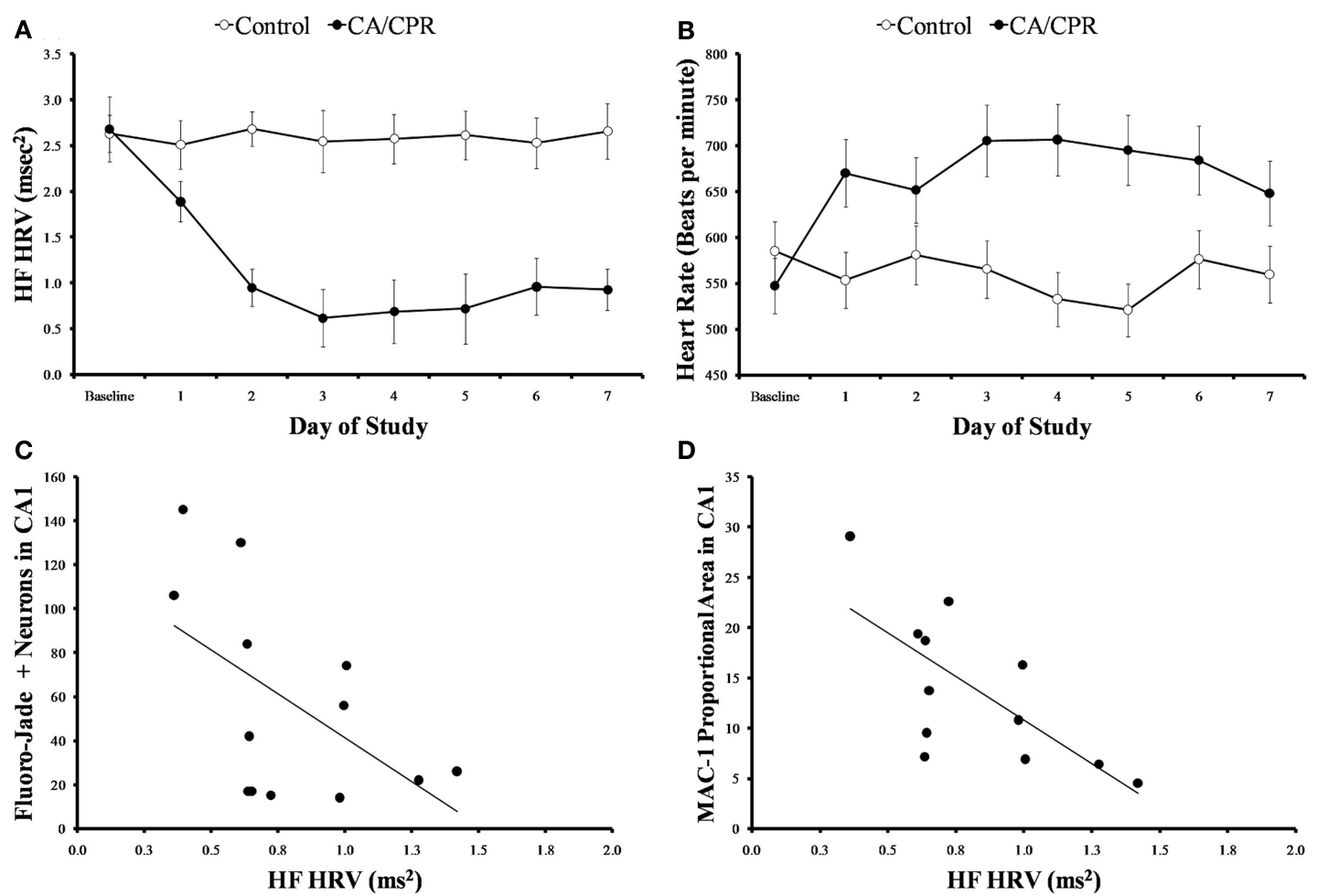

D

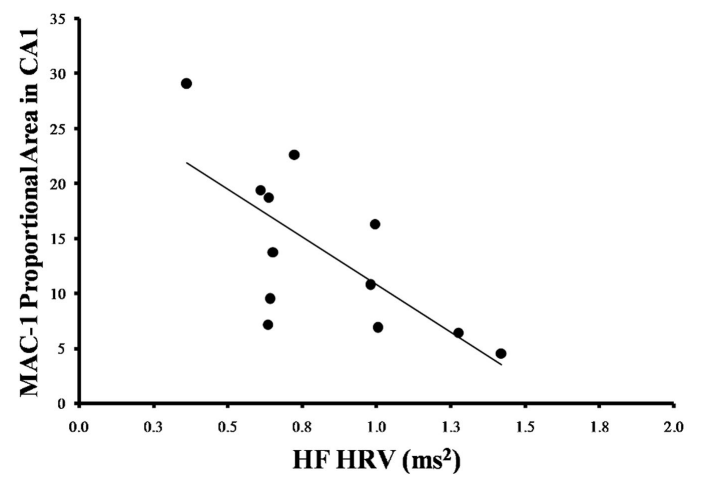

FIGURE 2 | (A) When cardiac arrest is accompanied by neurological damage (CA/CPR), there is a significant reduction in HF heart rate variability, a well validated measure of parasympathetic cardiac control. The mice in the ischemic control group (control) displayed similar levels of HF heart rate variability throughout the study. (B) Cardiac arrest also significantly increases heart rate. In contrast, the mice in the ischemic control group displayed

similar heart rates throughout the study. (C) At 7 days following normothermic CA/CPR, high frequency heart rate variability values were significantly and inversely correlated with measures of neuronal cell damage(c) and microglial activation (D) within the CA1 region of the hippocampus of mice. Data are presented as mean $\pm \mathrm{SD}$ and an asterisk indicates a significant difference $(p<0.05)$ at the given time point relative to the ischemic controls.

mice maintained typical diurnal rhythms in HF and LF heart rate variability $(p>0.05)$ and heart rate $(p>0.05)$ following CA/CPR. HF heart rate variability, and LF heart rate variability nor heart rate were significantly altered following CA/CPR in the hypothermic ischemic controls $(p>0.05)$.
Variations in HF heart rate variability at 7 days following surgery was significantly associated with the extent of cell death $\left(r_{12}^{2}=0.38, p<0.05\right.$, Figure $\left.2 \mathrm{C}\right)$ and microglia activation within the hippocampus $\left(r_{12}^{2}=0.34, p<0.05\right.$, Figure 2D). While LF heart rate variability was similarly correlated with cell death and 
microglia activation, the values did not reach statistical significance $(p>0.09)$. Heart rate, temperature, and activity were not associated with cell death or inflammatory processes following CA $(p>0.05)$. Furthermore, HF and LF heart rate variability were not significantly associated with alterations in temperature or activity $(p>0.05)$.

Furthermore, a 2 (light vs. dark) $\times 2$ (CA/CPR vs. control) ANOVA revealed that the CA/CPR procedure resulted in dysregulation of the diurnal rhythm in temperature $\left(F_{1,20}=6.05\right.$, $p<0.05 ;$ Figure 3A) and activity $\left(F_{1,20}=6.28, p<0.05\right.$; Figure 3B), Hypothermic control animals displayed comparable mean body temperature $(p>0.05)$ and activity levels $(p>0.05)$ throughout the baseline and post-surgical survival.

Cerebral ischemia resulted in acute hypothermia at $24-48 \mathrm{~h}$ following surgery $\left(F_{1,20}=6.52, p<0.05\right.$; Figure $\left.3 \mathrm{~A}\right)$ but returned to baseline levels by day 7 . Furthermore, cerebral ischemia increased overall locomotor activity within the animal's home cage $\left(F_{7,140}=8.02, p<0.05\right.$; Figure 3B $)$ as compared to the control group. Importantly, statistically controlling for locomotor behavior and temperature did not alter the outcome of neurocardiac functioning discussed above $(p>0.05)$; this observation suggests that the effects of cerebral ischemia on autonomic functioning are not the result of compensatory processes resulting from activity or temperature fluctuations.

\section{DISCUSSION}

As expected, the CA/CPR group sustained significantly greater neuronal damage and microglial activation within the hippocampus than the ischemic controls. Furthermore, global cerebral ischemia resulted in a rapid increase in heart rate, potentially the result of a decrease in parasympathetic cardiac control. An alternative explanation for the elevated heart rate could be that $\mathrm{CA} / \mathrm{CPR}$ results in an increase in sympathetic activity or intrinsic heart rate. Although we did not directly investigate the function of sympathetic nervous system function and intrinsic heart rate in the this study, previous work from our lab have discovered that the CA/CPR procedure results in significant decreases in sympathetic nervous system function and no changes in intrinsic heart rate by 7 days following surgery (Norman et al., 2010a). Specifically, the CA/CPR mice displayed significantly lower HF heart rate variability beginning $24 \mathrm{~h}$ following surgery and this pattern continued for 7 days following surgical manipulation. This is similar to a pattern reported in post MI human patients ( $\mathrm{La}$ Rovere et al., 1998; Berntson et al., 2008). The CA/CPR group also exhibited hyperactivity and acute hypothermia, however, these effects were independent of variations in parasympathetic cardiac control. While the CA/CPR procedure did not influence diurnal variations in $\mathrm{HF}$ heart rate variability, $\mathrm{LF}$ heart rate variability or heart rate, it did significantly decrease diurnal rhythmicity in temperature and activity, which also has been reported after experimental stroke (Karelina et al., 2009). Thus, the neuroinflammation and neuronal damage caused by CA is associated with severe dysregulation of parasympathetic cardiac control, which is associated with poorer health outcomes in humans (Thayer and Lane, 2007). Although causal influences of neuronal cell death on parasympathetic cardiac control cannot be determined in the present study, one potential explanation for this association may be alterations in central cholinergic signaling. Indeed, previous work has demonstrated that central cholinergic signaling regulates autonomic nervous system functioning (Berntson et al., 1994) and $\mathrm{CA} / \mathrm{CPR}$ results in decreases in basal forebrain choline acetyltransferase (ChAT positive neurons and central ChAT activity (Norman et al., 2010b). ChAT is an important enzyme responsible for the synthesis of acetylcholine. Furthermore, cholinergic neurons have been shown to be highly susceptible to the deleterious effects of neuroinflammation (Wenk and Willard, 1998). Future studies will be necessary in order to determine whether the results presented in this manuscript are a function of neuroinflammation induced toxicity of cholinergic neurons.

Measurement of autonomic function is widely used in clinical settings as a predictor of mortality in chronic heart disease patients (Malik, 1996; Sans et al., 1997). However, despite the strong predictive value of heart rate variability, the relationship between low heart rate variability and increased mortality is not well understood. The significant inverse correlation between heart rate variability and neuronal damage in this study suggests that low heart rate variability could be an indicator of brain damage following CA (Figures 2C,D). The hippocampus is one of the most susceptible regions of the brain to ischemic damage (Kofler et al., 2004; Neigh et al., 2009), and directly innervates
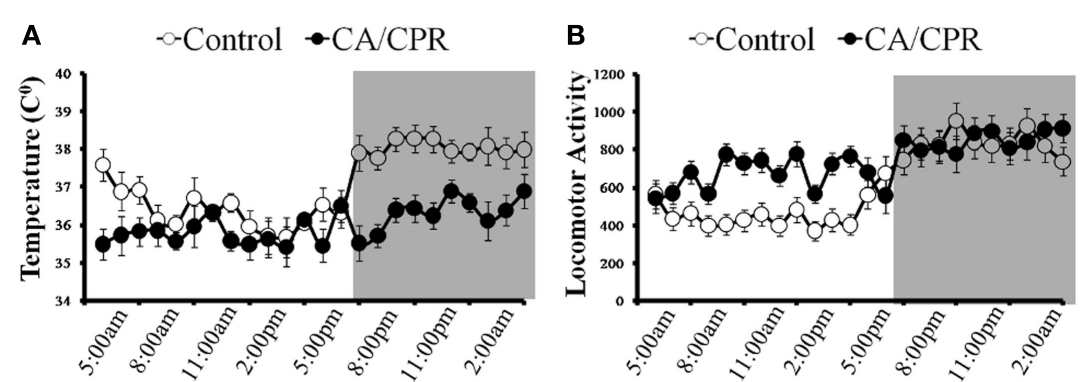

FIGURE 3 | (A) When cardiac arrest is accompanied by neurological damage (CA/CPR), there is a significant reduction in body temperature and dysregulation of the diurnal rhythm. In contrast, body temperature and its diurnal rhythm did not differ from baseline among the ischemic control group that did not sustain neurological damage. (B) Likewise, the CA/CPR group exhibited increased locomotor behavior and dysregulation of the diurnal rhythm in activity, whereas locomotor activity and diurnal rhythmicity in activity did not vary throughout the study among the ischemic control mice. Data are collapsed across post-surgical days 1-7. The dark background denotes lights off (animal's active period). Data are presented as mean \pm SD. 
numerous structures included within the central autonomic network; the central autonomic network includes the prefrontal cortex, hypothalamus, amygdala, periaqueductal gray, the nucleus of the solitary tract, the nucleus ambiguous, the ventral medulla, and medullary tegmental field which together modulate the parasympathetic nervous system (Thayer and Lane, 2007). Indeed, direct hippocampal stimulation decreases heart rate, and respiratory rate, a result blocked by methylatropine indicating vagally mediated changes in heart rate may be partially mediated by the hippocampus (Ruit and Neafsey, 1988). The ability of the hippocampus to modify the central autonomic network provides a potential mechanism through which measurement of autonomic functioning can provide rapid and reliable information on the extent of neuronal damage following CA.

Monitoring the autonomic nervous system after cerebral ischemia also may provide information regarding ongoing neuroinflammatory processes. Following CA, there is a significant inverse correlation between heart rate variability and microglial activation (Figure 2D). Microglia are resident immune cells of the central nervous system that survey the environment and become rapidly activated in response to CNS injury; microglial activation, which prompts the release of proinflammatory cytokines, represents the first phase of the CNS inflammatory response to cerebral ischemia (Yenari et al., 2010). Likewise, heart rate variability is inversely related to concentrations of proinflammatory cytokines and other inflammatory markers in the blood of healthy adults, as well as those with cardiovascular disease (Sloan et al., 2007; Haensel et al., 2008). In the current study, it is not possible to determine whether a causal relationship exists between CA-induced neuroinflammation and the reduction in heart rate variability, although the literature supports effects in both directions. For example, inducing cytokine expression in healthy adults via administration of an endotoxin results in a rapid reduction in heart rate variability (Marsland et al., 2007; Kox et al., 2011). Similarly, cytokine concentrations and mortality are inversely related to heart rate variability in mice exposed to live bacteria (Fairchild et al., 2011). Alternatively, diminished parasympathetic nervous

\section{REFERENCES}

American Heart Association. (2002). Heart Disease and Stroke Statistics: 2003 Update. Dallas, TX: American Heart Association.

Berntson, G. G., Bigger, J. T. Jr., Eckberg, D. L., Grossman, P., Kaufmann, P. G., Malik, M., Nagaraja, H. N., Porges, S. W., Saul, J. P., Stone, P. H., and van der Molen, M. W. (1997). Heart rate variability: origins, methods, and interpretive caveats. Psychophysiology 34, 623-648.

Berntson, G. G., Cacioppo, J. T., Binkley, P. F., Uchino, B. N., Quigley, K. S., and Fieldstone, A. (1994). Autonomic cardiac control. III. Psychological stress and cardiac response in autonomic space as revealed by pharmacological blockades. Psychophysiology 31, 599-608.

Berntson, G. G., Norman, G. J., Hawkley, L. C., and Cacioppo,
J. T. (2008). Cardiac autonomic balance versus cardiac regulatory capacity. Psychophysiology 45, 643-652.

Bigger, J. T. Jr., Kleiger, R. E., Fleiss, J. L., Rolnitzky, L. M., Steinman, R. C., and Miller, J. P. (1988). Components of heart rate variability measured during healing of acute myocardial infarction. Am. J. Cardiol. 61, 208-215.

Cronberg, T., Lilja, G., Rundgren, M., Friberg, H., and Widner, H. (2009). Long-term neurological outcome after cardiac arrest and therapeutic hypothermia. Resuscitation 80, 1119-1123.

Fairchild, K. D., Srinivasan, V., Moorman, J. R., Gaykema, R. P., and Goehler, L. E. (2011). Pathogen-induced heart rate changes associated with cholinergic nervous system activation. Am. J.

system activity can lead to "run away" inflammation (Tracey, 2009). Indeed, CA impairs turn diminishes the capacity of the cholinergic anti-inflammatory pathway to control inflammation after cerebral ischemia; pharmacological activation of $\alpha 7$ nicotinic acetylcholine receptors within $24 \mathrm{~h}$ of CA provides significant protection against ischemia-related cell death and inflammation (Norman et al., 2010b). Thus, in conjunction with its association with pathophysiological responses to $\mathrm{CA} / \mathrm{CPR}$, diminished parasympathetic output may directly influence health outcomes through a diminished capacity to regulate cardiovascular and inflammatory processes.

There are limitations to the present study that should be acknowledged. Firstly, the present study analyzed histological outcome at a single time point ( 7 days post surgery). While this time point is commonly used in ischemia model research, future studies will need to determine whether the relationships described in this manuscript are apparent at later time points. Furthermore, one is not able to infer a causal relationship between heart rate variability from the results presented in this manuscript. It will be important for future studies to determine whether the neuronal damage and inflammation presented here is causally related to lower heart rate variability or whether lower heart rate variability increases susceptibility to neuronal damage following CA/CPR.

In sum, HF heart rate variability is associated with the extent of cell death and inflammation that occurs in the brain following CA, suggesting that non-invasive measurement of autonomic functioning could serve as a functional marker of the neural effects of cerebral ischemia. The inverse linear relationships between heart rate variability and brain damage after CA also may partially explain why low heart rate variability is associated with increased morbidity and mortality in myocardial infarction patients; patients with low heart rate variability may have more extensive brain damage, which in turn compromises survival. As more treatments become available for CA survivors, monitoring HF heart rate variability may be a rapid, non-invasive, and inexpensive method for assessing efficacy in preserving the brain.

Physiol. Regul. Integr. Comp. Physiol. 300, R330-R339.

Gehrmann, J., Hammer, P. E., Maguire, C. T., Wakimoto, H., Triedman, J. K., and Berul, C. I. (2000). Phenotypic screening for heart rate variability in the mouse. Am. J. Physiol. Heart Circ. Physiol. 279, H733-H740.

Haensel, A., Mills, P. J., Nelesen, R. A., Ziegler, M. G., and Dimsdale, J. E. (2008). The relationship between heart rate variability and inflammatory markers in cardiovascular diseases. Psychoneuroendocrinology 33, 1305-1312.

Huikuri, H. V., Raatikainen, M. J., Moerch-Joergensen, R., Hartikainen, J., Virtanen, V., Boland, J. Anttonen, O., Hoest, N., Boersma, L. V., Platou, E. S., Messier, M. D., and Bloch-Thomsen, P. E. (2009). Cardiac Arrhythmias and risk stratification after acute myocardial infarction study group. Eur. Heart J. 30, 689-698.

Karelina, K., Norman, G. J., Zhang, N., and DeVries, A. C. (2009). Social contact influences histological and behavioral outcomes following cerebral ischemia. Exp. Neurol. 220, 276-282.

Karp, E., Shiyovich, A., Zahger, D., Gilutz, H., Grosbard, A., and Katz, A. (2009). Ultra-short-term heart rate variability for early risk stratification following acute ST-elevation myocardial infarction. Cardiology 114, 275-283.

Kofler, J., Hattori, K., Sawada, M., DeVries, A. C., Martin, L. J., Hurn, P. D., and Traystman, R. J. (2004). Histopathological and behavioral characterization of a novel model of cardiac arrest and cardiopulmonary resuscitation in mice. J. Neurosci. Methods 136, 33-44. 
Kox, M., Ramakers, B. P., Pompe, J. C., van der Hoeven, J. G., Hoedemaekers, C. W., and Pickkers, P. (2011). Interplay between the acute inflammatory response and heart rate variability in healthy human volunteers. Shock 36, 115-120.

La Rovere, M. T., Bigger, J. T. Jr., Marcus, F. I., Mortara, A., and Schwartz, P. J. (1998). Baroreflex sensitivity and heart-rate variability in prediction of total cardiac mortality after myocardial infarction. ATRAMI (Autonomic Tone and Reflexes after Myocardial Infarction) Investigators. Lancet 351, 478-484.

Malik, M. (1996). Heart rate variability: standards of measurement, physiological interpretation and clinical use. Task Force of the European Society of Cardiology and the North American Society of Pacing and Electrophysiology. Circulation 93, 1043-1065.

Marsland, A. L., Gianaros, P. J., Prather, A. A., Jennings, J. R., Neumann, S. A., and Manuck, S. B. (2007). Stimulated production of proinflammatory cytokines covaries inversely with heart rate variability. Psychosom. Med. 69, 709-716.

Micieli, G., and Cavallini, A. (2008). The autonomic nervous system and ischemic stroke: a reciprocal interdependence. Clin. Auton. Res. 18, 308-317.

Neigh, G. N., Karelina, K., Zhang, N., Glasper, E. R., Owens, M. J., Plotsky, P. M., Nemeroff, C. B., and Devries,
A. C. (2009). Cardiac arrest and cardiopulmonary resuscitation dysregulates the hypothalamic-pituitaryadrenal axis. J. Cereb. Blood Flow Metab.

Norman, G. J., Berntson, G. G., Morris, J. S., Karelina, K., Zhang, N., Weil, Z. M., and DeVries, A. C. (2010a). Social isolation exacerbates autonomic, inflammatory and behavior responses to global cerebral ischemia. Proc. Natl. Acad. Sci. U.S.A. 107, 16342-16347.

Norman, G. J., Karelina, K., Berntson, G. G., Weil, Z. M., Zhang, N., Morris, J. S., Al-Abed, Y., Pavlov, V. A., Tracey, K. J., and DeVries, A. C. (2010b). Cardiopulmonary arrest and resuscitation disrupts cholinergic antiinflammatory processes: a role for cholinergic $\alpha 7$ nicotinic receptors. J. Neurosci. 31, 3446-3452.

Offner, H., Vandenbark, A. A., and Hurn, P. D. (2009). Effect of experimental stroke on peripheral immunity: CNS ischemia induces profound immunosuppression. Neuroscience 158, 1098-1111.

Oppenheimer, S. M., Kedem, G., and Martin, W. M. (1996). Left-insular cortex lesions perturb cardiac autonomic tone in humans. Clin. Auton. Res. 6, 131-140.

Popovich, P. G., Wei, P., and Stokes, B. T. (1997). Cellular inflammatory response after spinal cord injury in Sprague-Dawley and Lewis rats. J. Comp. Neurol. 377, 443-464.

Ruit, K. G., and Neafsey, E. J. (1988). Cardiovascular and respiratory responses to electrical and chemical stimulation of the hippocampus in anesthetized and awake rats. Brain Res. 457, 310-321.

Sans, S., Kesteloot, H., and Kromhout, D. (1997). The burden of cardiovascular diseases mortality in Europe. Task Force of the European Society of Cardiology on Cardiovascular Mortality and Morbidity Statistics in Europe. Eur. Heart J. 18, 1231-1248.

Schmued, L. C., and Hopkins, K. J. (2000). Fluoro-Jade B: a high affinity fluorescent marker for the localization of neuronal degeneration. Brain Res. 874, 123-130.

Sloan, R. P., McCreath, H., Tracey, K. J., Sidney, S., Liu, K., and Seeman, T. (2007). RR interval variability is inversely related to inflammatory markers: The CARDIA study. Mol. Med. 13, 178-184.

Thayer, J. F., Hansen, A. L., Saus-Rose, E. and Johnsen, B. H. (2009). Heart rate variability, prefrontal neural function, and cognitive performance: the neurovisceral integration perspective on self-regulation, adaptation, and health. Ann. Behav. Med. 37, 122-129.

Thayer, J. F., and Lane, R. D. (2007) The role of vagal function in the risk for cardiovascular disease and mortality. Biol. Psychol. 74, 224-242.

Thireau, J., Zhang, B. L., Poisson, D. and Babuty, D. (2008). Heart rate variability in mice: a theoretical and practical guide. Exp. Physiol. 93, 83-94.

Tracey, K. J. (2009). Reflex control of immunity. Nat. Rev. Immunol. 9, 418-428.
Wenk, G. L., and Willard, L. B. (1998). The neural mechanisms underlying cholinergic cell death within the basal forebrain. Int. J. Dev. Neurosci. 16, 729-735.

Yenari, M. A., Kauppinen, T. M., and Swanson, R. A. (2010). Microglial activation in stroke: therapeutic targets. Neurotherapeutics 7, 378-391.

Conflict of Interest Statement: The authors declare that the research was conducted in the absence of any commercial or financial relationships that could be construed as a potential conflict of interest.

Received: 02 October 2011; accepted: 19 April 2012; published online: 10 May 2012.

Citation: Norman GJ, Karelina K, Berntson GG, Morris JS, Zhang $N$ and DeVries AC (2012) Heart rate variability predicts cell death and inflammatory responses to global cerebral ischemia. Front. Physio. 3:131. doi: 10.3389/fphys.2012.00131

This article was submitted to Frontiers in Integrative Physiology, a specialty of Frontiers in Physiology.

Copyright (C) 2012 Norman, Karelina, Berntson, Morris, Zhang and DeVries. This is an open-access article distributed under the terms of the Creative Commons Attribution Non Commercial License, which permits non-commercial use, distribution, and reproduction in other forums, provided the original authors and source are credited. 